\title{
A survey of labour epidural practices at obstetric anesthesia fellowship programs in the United States
}

\section{Un sondage sur les pratiques de péridurales pour le travail dans les programmes de fellowship en anesthésie obstétricale aux États- Unis}

\author{
Elliott Callahan, MD (iD - Peter Yeh, MD • Brendan Carvalho, MD • \\ Ronald B. George, MD, FRCPC
}

Received: 1 October 2021/Revised: 16 November 2021/ Accepted: 17 November 2021/Published online: 28 January 2022

(C) The Author(s) 2022

\begin{abstract}
Purpose Labour epidural analgesia (LEA) is an evolving field. Various neuraxial techniques and dosing regimens are available to the modern obstetric anesthesia provider, allowing for significant practice variability. To begin a search for consensus on optimal care, we sought to query fellowship training practices for LEA.

Methods We conducted an electronic survey of institutions with American Council for Graduate Medical Education-accredited obstetric anesthesiology fellowship programs. We studied the frequency of epidural initiation techniques, including combined spinal epidural (CSE), dural puncture epidural, and epidural bolus. For maintenance techniques, we appraised the use of continuous epidural infusion, programmed intermittent bolus (PIEB), and patient-controlled epidural analgesia (PCEA).
\end{abstract}

Results of 40 institutions surveyed, we received 32 responses ( $80 \%$ response rate). Twenty-eight of 40 (70\%) were included in the analysis. A plurality of institutions $(12 / 28 ; 43 \%)$ preferred CSE, and among those who used

Supplementary Information The online version contains supplementary material available at https://doi.org/10.1007/s12630$022-02192-6$

E. Callahan, MD $(\varangle) \cdot$ P. Yeh, MD

R. B. George, MD, FRCPC

Department of Anesthesia and Perioperative Care, University of California San Francisco (UCSF), 513 Parnassus Ave, MSB,

436, Box 0427, San Francisco, CA 94143, USA

e-mail: elliott.callahan@ucsf.edu

B. Carvalho, MD

Stanford University, Palo Alto, CA, USA
CSE, 23/27 (85\%) included intrathecal opioids. A majority of institutions used protocols with PIEB (55\%), while almost all (92\%) used PCEA. Most participants (88\%) reported using dilute concentration maintenance infusions of $0.1 \%$ bupivacaine/ropivacaine or less.

Conclusion Despite significant variability in LEA practice, some clear patterns emerged in our survey, including preference for opioid-containing CSE and maintenance with PIEB, PCEA, and dilute epidural solutions.

\section{Résumé}

Objectif L'analgésie péridurale obstétricale (APO) est un domaine en évolution. Diverses techniques neuraxiales et posologies sont disponibles pour le praticien en anesthésie obstétricale moderne, ce qui permet une variabilité significative de la pratique. Pour démarrer une recherche de consensus sur les soins optimaux, nous avons cherché à déterminer les pratiques d'APO dans le cadre de formation des fellows.

Méthode Nous avons réalisé une enquête électronique auprès d'établissements possédant des programmes de fellowship en anesthésiologie obstétricale accrédités par l'American Council for Graduate Medical Education. Nous avons étudié la fréquence des techniques de péridurale, notamment de rachi-péridurale combinée (RPC), de péridurale avec ponction durale et de bolus péridural. Pour les techniques de maintien, nous avons évalué l'utilisation de l'analgésie péridurale par perfusion continue, l'administration programmée de bolus périduraux (PIEB; programmed intermittent epidural bolus) et l'analgésie péridurale contrôlée par la patiente (APCP). 
Résultats Sur les 40 établissements sondés, nous avons reçu 32 réponses (taux de réponse de $80 \%$ ). Vingt-huit des $40(70 \%)$ établissements ont été inclus dans l'analyse. Plusieurs institutions (12/28; $43 \%)$ ont répondu qu'elles préféraient la RPC, et parmi celles qui utilisaient la RPC, 23/27 (85\%) incluaient des opioïdes intrathécaux. La majorité des établissements utilisaient des protocoles avec le PIEB (55\%), tandis que presque tous (92\%) utilisaient l'APCP. La plupart des établissements participants (88\%) ont rapporté utiliser des perfusions de maintien à des concentrations diluées de bupivacaïne/ropivacaïne de 0,1 $\%$ ou moins.

Conclusion Malgré une variabilité significative dans la pratique de l'APO, certaines tendances claires ont émergé dans notre sondage, notamment une préférence pour les $R P C$ contenant des opioïdes et le maintien avec un PIEB, une APCP et des solutions péridurales diluées.

Keywords labour · neuraxial · protocol · survey · fellowships

Labour epidural analgesia (LEA) is widely regarded as the most effective means of providing pain relief during labour. Nevertheless, the optimal LEA technique is still controversial. Several variations in standard epidural placement techniques are described, differing in whether the placement of the epidural catheter is preceded by puncture of the dura mater with a spinal needle-dural puncture epidural (DPE) - and whether that needle is used to inject intrathecal (IT) medication, performing what is termed a combined spinal epidural (CSE). Further practice variation then arises as to whether a bolus of medication is delivered through the catheter into the epidural space to initiate analgesia. When these techniques are multiplied by all possible analgesics that may be used in the epidural or IT space, the result is a myriad of initiation options, leading to great potential for practice heterogeneity. ${ }^{1,2}$

Historically, practices centred around continuous epidural infusion (CEI) of local anesthetic (LA). Patientcontrolled epidural bolus (PCEA) with or without CEI was then introduced for breakthrough pain. ${ }^{3}$ Recently, there has been an increasing body of evidence supporting the use of programmed intermittent epidural bolus (PIEB) as a technique to improve maternal satisfaction scores, reduce total drug use, and reduce provider workloads. ${ }^{4-6}$ Nevertheless, the prevalence of PIEB adoption has not been well studied, and practice variation has not been evaluated.

Our study aimed to assess contemporary LEA practices at leading academic obstetric anesthesia institutions. We chose to survey fellowship training programs because we reasoned they would represent up-to-date practice. In so doing, we sought to identify areas of consensus and variation among anesthesia providers about what constitutes optimal LEA care, as imparted to future obstetric anesthesiologists.

\section{Methods}

We developed a 31-question electronic questionnaire using the University of California San Francisco Qualtrics survey platform, which was then distributed to anesthesiologists who are program directors of American Council for Graduate Medical Education (ACGME)-accredited obstetric anesthesiology fellowships. The survey queried demographic information about each institution (including estimations of the number of births and epidurals and mode of delivery rates), types of anesthesia/analgesia provided (including pharmacologic interventions and estimated frequencies), and how these are administered. The survey was developed by a team of board-certified obstetric anesthesiologists and an anesthesiology resident.

After obtaining institutional review board approval (UCSF IRB \#20-32908; 20 January 2021), we used publicly available contact information to email a survey invitation to program directors. The email contained background information on the study with an online link to the questionnaire. A modified Dillman approach was then used to remind and encourage participation. ${ }^{7}$ An introductory email was sent on 25 February 2021, and the survey opened on 1 March 2021 and closed on 11 April 2021. Participation was incentivized with entry into a draw for one of two USD 100 Amazon gift cards. Response data were downloaded from the Qualtrics application into a Microsoft Excel (Microsoft Corporation, Redmond, WA, USA) file for data analysis. We analyzed types of LEA technique using descriptive statistics in RStudio desktop software (version 1.4.1717, RStudio PBC, Boston, MA, USA).

\section{Results}

Out of a total of 40 survey requests sent, 32 unique responses were received (80\% response rate) and 28/32 (70\%) surveys were included in the analysis (Electronic Supplementary Material [ESM] eFig. 1). Seventeen states in the USA were represented in the responses (ESM eFig. 2). Among the represented institutions, the number of annual live births ranged from 2,000 to 11,500 , with a median [interquartile range (IQR)] of 4,350 [3,500-6,000] births. The rate of parturients receiving LEA ranged from $72 \%$ to $95 \%$, with a median rate of $85 \%$. The Cesarean 
delivery rate ranged from $22 \%$ to $50 \%$ with a median rate of $30 \%$, and the median rate of instrumented vaginal delivery (e.g., vacuum or forceps-assisted) as a percentage of all deliveries was $5 \%$.

\section{Labour epidural analgesia initiation}

Among the institutions we surveyed, there was a preference to initiate with CSE (median frequency, 34\%) over DPE $(20 \%)$, or standard epidural bolus (EPI; 18\%); however, a wide range of practice was reported (Fig. 1). Twenty-six institutions had a "preferred" method of analgesia initiation (Table 1): $43 \%$ (12/28) preferred CSE, $21 \%$ preferred DPE (6/28), and 29\% (8/28) preferred EPI. Seven percent of institutions (2/28) did not have a preferred method of initiation. Among the institutions who reported the LA used for CSE (26/28), isobaric bupivacaine was used by 24 (92\%). The median (range) amount of bupivacaine administered was 2.5 (1.25-3.75) $\mathrm{mg}$. Two respondents reported using IT ropivacaine.

Twenty-seven of 28 respondents reported performing CSE, and 23/27 (85\%) included an opioid in the CSE spinal dose. The commonest opioid among those was fentanyl $(21 / 23 ; 91 \%)$; the median (range) and commonest dose thereof being $15(4-25) \mu \mathrm{g}(n=9)$. The two respondents who used sufentanil reported doses of 5 and $6 \mu \mathrm{g}$, respectively.

For institutions that used PIEB, we asked the time interval from CSE to the first PIEB dose, defined as the "next bolus". Next bolus times ranged from 0 to $45 \mathrm{~min}$, while the most selected delay was $30 \mathrm{~min}$ (mode/median, 30).
For institutions that performed EPI (with or without DPE) in lieu of CSE, the commonest LA was bupivacaine (89\% of respondents; Table 2). The commonest concentration used was $0.125 \%$ (50\% of respondents). Among those who used ropivacaine (11\% of respondents), the most commonly administered concentration was $0.1 \%$. Twenty-one percent (6/28) of all respondents bolused with the same solution used for maintenance. The commonest initiation volume administered-regardless of LA chosen-was $10 \mathrm{~mL}$ (range, 5-20 mL). The median [IQR] (range) initiation bolus volume was 10 [10-10] (515) $\mathrm{mL}$ for DPE and 10 [10-15] (10-20) $\mathrm{mL}$ for EPI. Of the respondents, 68\% (19/28) included an opioid in their initiation bolus, most commonly fentanyl (57\%). The most common dose of fentanyl was $100 \mu \mathrm{g}$ (range, 5-100 $\mu \mathrm{g}$ ). Among those who used sufentanil (11\%), the median (range) dose was $15(5-20) \mu$ g. Table 2 summarizes the preferred choices for epidural bolus administration among participating institutions in aggregate form. Individual institutions' preferred initiation techniques are listed in ESM eTable 2.

\section{Labour epidural analgesia maintenance}

Twenty-seven of 28 institutions (96\%) had a standardized maintenance protocol. A full, detailed list of all participating institutions' individual maintenance regimens is presented in ESM eTable 2. Fourteen unique combinations of LA and opioid solutions were reported. We excluded two responses: one reporting "bupivacaine $0.8 \%$ "-assumed to be erroneous-and one reporting " $0.08325 \%$ " of an unspecified LA, for a total of 26 analyzed regimens. The most common LA used was

Frequency Using Initiation Technique

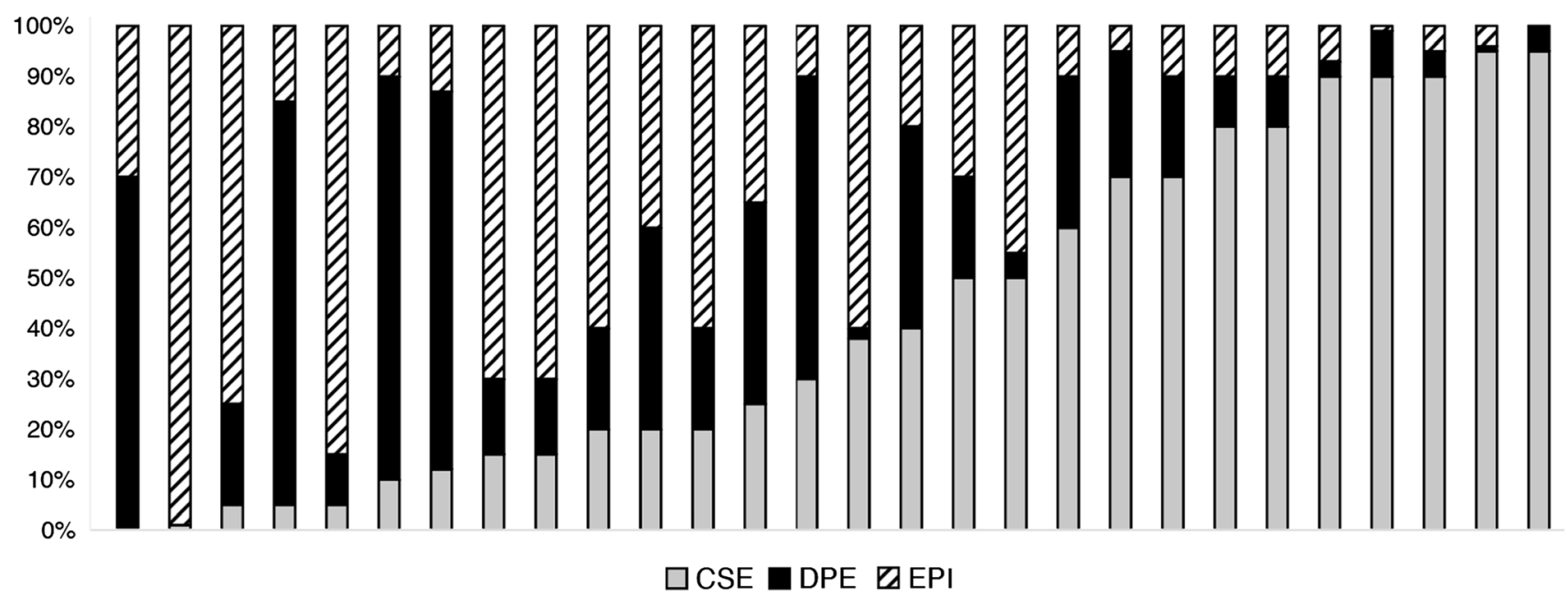

Fig. 1 Frequency of initiation techniques across participating institutions. Each column represents an individual participating obstetric anesthesia fellowship program. 
Table 1 Preferred choices for epidural initiation

\section{CSE}

Preferred, $n /$ total $N(\%) \quad 12 / 28(43 \%)$

\begin{tabular}{|c|c|c|c|c|c|}
\hline & & Prevalence, $\%(n /$ total $N)$ & Dose (mg), median [IQR] & Dose $(\mathrm{mg})$, mode & Dose $(\mathrm{mg})$, range \\
\hline \multirow[t]{2}{*}{ LA } & Isobaric bupivacaine & $92 \%(24 / 26)^{\mathrm{a}}$ & $2.5[1.75-2.5]$ & 2.5 & $1.25-3.75$ \\
\hline & Ropivacaine & $8 \%(2 / 26)^{\mathrm{a}}$ & 2 & & 2 \\
\hline \multirow[t]{3}{*}{ Opioid } & Any opioid & $85 \%(23 / 27)^{\mathrm{b}}$ & & & \\
\hline & Fentanyl & $91 \%(21 / 23)^{\mathrm{c}}$ & $15[10-15]$ & 15 & $4-25$ \\
\hline & Sufentanil & $9 \%(2 / 23)^{\mathrm{c}}$ & 5.5 & & $5-6$ \\
\hline $\mathbf{L}$ & & & & & \\
\hline Preferred, $n /$ total $N(\%)$ & $6 / 28(21 \%)$ & & & & \\
\hline EPP & & & & & \\
\hline eferred, $n /$ total $N(\%)$ & $8 / 28(29 \%)$ & & & & \\
\hline
\end{tabular}

Sample sizes and percentages (n/total $N[\%])$ reflect institutions

${ }^{a}$ Among those who use a local anesthetic

${ }^{\mathrm{b}}$ Among those who perform CSE

${ }^{c}$ Among those who use any opioid

$\mathrm{CSE}=$ combined spinal-epidural, $\mathrm{DPE}=$ dural puncture epidural, $\mathrm{EPI}=$ epidural bolus (without DPE), IQR $=$ interquartile range, $\mathrm{LA}=$ local anesthetic

Table 2 Preferred choices for epidural bolus administration (with or without dural puncture epidural technique)

\begin{tabular}{|c|c|c|c|c|}
\hline \multirow[t]{9}{*}{ LA, $n /$ total $N(\%)$} & Bupivacaine $0.125 \%$ & $14 / 28(50 \%)$ & & \\
\hline & Bupivacaine $0.25 \%$ & $4 / 28(14 \%)$ & & \\
\hline & Bupivacaine $0.1 \%$ & $1 / 28(4 \%)$ & & \\
\hline & Bupivacaine $0.0625 \%$ & $4 / 28(14 \%)$ & & \\
\hline & Bupivacaine $0.04 \%$ & $2 / 28(7 \%)$ & & \\
\hline & Bupivacaine total & $25 / 28(89 \%)$ & & \\
\hline & Ropivacaine $0.15 \%$ & $1 / 28(4 \%)$ & & \\
\hline & Ropivacaine $0.1 \%$ & $2 / 28(7 \%)$ & & \\
\hline & Ropivacaine total & $3 / 28(11 \%)$ & & \\
\hline \multirow[t]{2}{*}{ LA volume $(\mathrm{mL})$} & Median [IQR] & Range & & \\
\hline & $10[10-15]$ & $5-20$ & & \\
\hline \multirow[t]{3}{*}{ Opioid, $n /$ total $N(\%)$} & Any opioid & $19 / 28(68 \%)$ & & \\
\hline & Fentanyl & $16 / 28(57 \%)$ & & \\
\hline & Sufentanil & $3 / 28(11 \%)$ & & \\
\hline \multirow[t]{3}{*}{ Opioid dose $(\mu \mathrm{g})$} & & Median [IQR] & Mode & Range \\
\hline & Fentanyl & $33[20-81]$ & 100 & $5-100$ \\
\hline & Sufentanil & $15[11-18]$ & & $6-20$ \\
\hline
\end{tabular}

Sample sizes and percentages (n/total $N[\%])$ reflect institutions

$\mathrm{IQR}$ = interquartile range

bupivacaine $0.0625 \% \quad(50 \%$ of respondents; 13/26). Overall, $88 \%(n=23 / 26)$ of included responses reported using concentrations of $\leq 0.1 \%$ bupivacaine/ropivacaine or less (Table 1).

Ninety-three percent (26/28) of respondents included an opioid in their maintenance solution, the most common of which was fentanyl $2 \mu \mathrm{g} \cdot \mathrm{mL}^{-1}$. Overall, the most prevalent solution used for maintenance was bupivacaine $0.0625 \%$ with fentanyl $2 \mu \mathrm{g} \cdot \mathrm{mL}^{-1}$, at $39 \%(11 / 28)$.

Twenty-seven institutions had a maintenance technique they used a majority of the time, which we designated their "preferred" maintenance method. Preferred methods are 
outlined in Fig. 2A. Patient-controlled epidural analgesia was nearly universally used $(93 \% ; 25 / 27)$. There was a slight preference for PIEB regimens over CEI (56\% vs $44 \%$ ).

Among all those who used CEI with PCEA, the median (range) infusion rate was $10(6-15) \mathrm{mL} \cdot \mathrm{hr}^{-1}$, the median (range) PCEA volume was 5 (3-10) $\mathrm{mL}$, and the median (range) lockout time was 15 (8-20) min. For institutions using PIEB with PCEA, the median (range) PIEB volume was 9 (5-10) $\mathrm{mL}$, and the commonest (mode) PIEB interval was $45 \mathrm{~min}$ (range, 30-60 min). The commonest PCEA lockout time was ten minutes (range, 10-15 min). There was a correlation between larger PIEB volumes and longer intervals $(P=0.002)$; however, there was no statistically significant trend between PCEA volume and lockout, or PIEB volume and PCEA volume. Figure 2B shows CSE, DPE, or EPI stratified by maintenance method, showing a predominance of CSE among institutions who also use PIEB.

\section{Discussion}

We conducted an exploratory survey of LEA practices at twenty-eight US institutions with ACGME-accredited obstetric anesthesiology fellowships to identify consensus about what constitutes standard LEA care and to determine variations among leading obstetric anesthesia providers. Our response rate $(80 \%)$ was excellent compared with studies of surveys directed at anesthesiologists, which can average as low as $37 \% .^{8}$

Our results show that, among placement/initiation techniques, there was a preference for CSE over DPE or simple EPI. Among those who used CSE, the vast majority included an opioid in the IT mixture. We did not investigate standardized initiation protocols, but there was significant inter- and intrainstitutional variability to suggest that few exist (Fig. 1). This is likely due to many factors, including vital signs at time of initiation, fetal heart rate tracing category, or expected maintenance technique.
Fig. 2 Preferred maintenance methods and initiation methods based on maintenance regimens
A

Preferred Maintenance Method

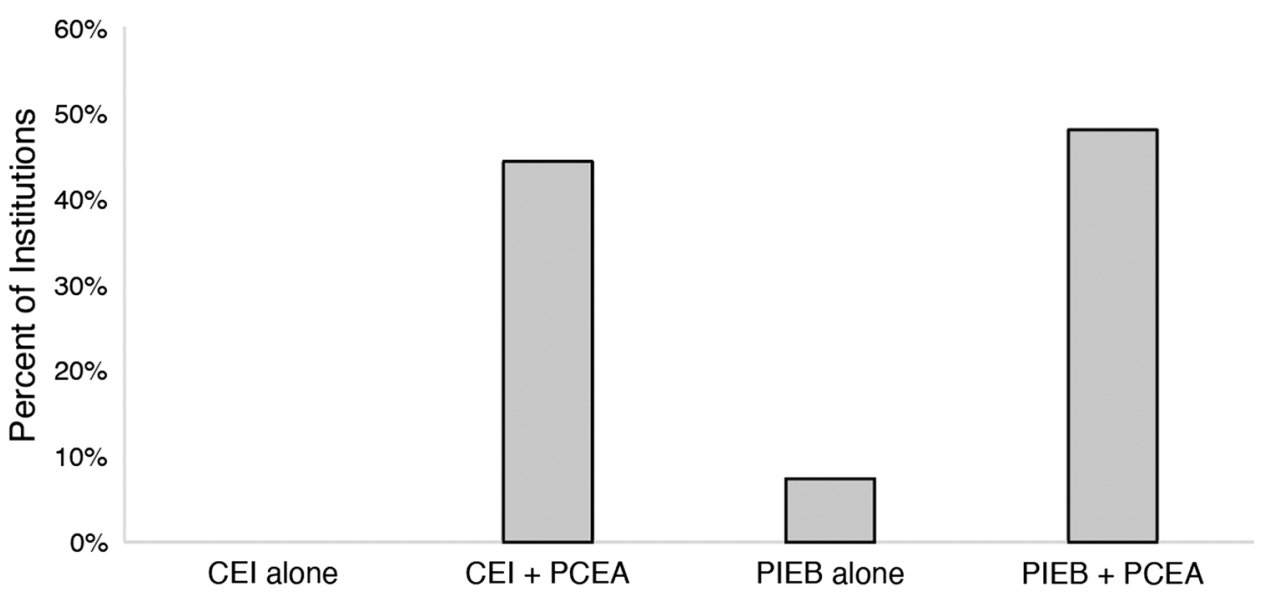

B

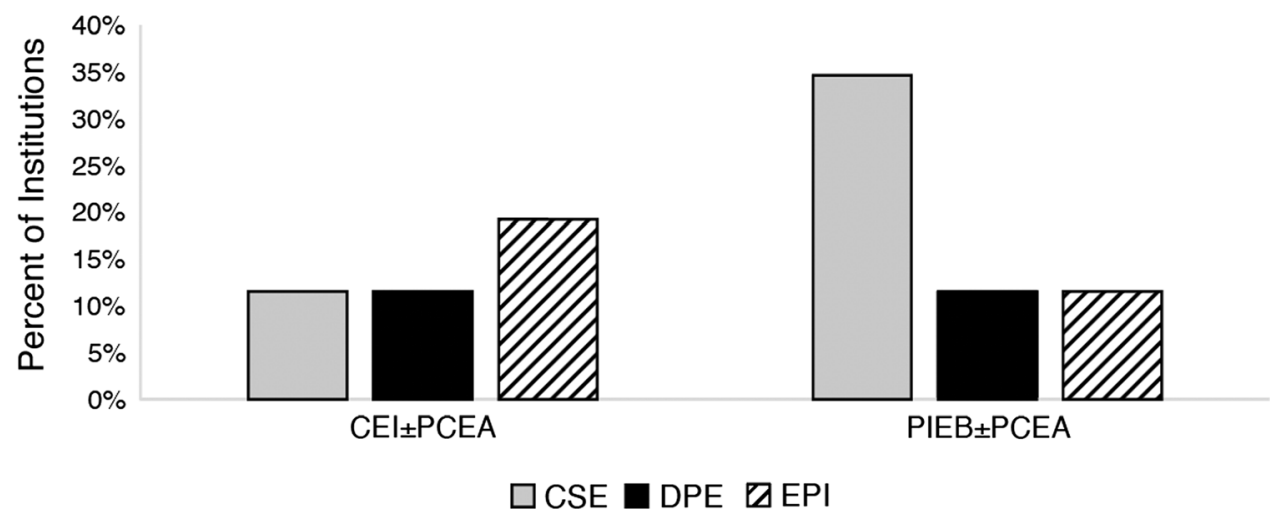


Among maintenance regimens, we found that essentially no institution relied on CEI alone, respecting strong evidence that inclusion of PCEA functionality confers significant advantages, including reduced motor block and reduced clinician interventions. ${ }^{9}$ The one respondent who reported using CEI alone more than $5 \%$ of the time specified that they were in the process of transitioning to a PIEB with PCEA model within the year. Other respondents specified that CEI was reserved for "walking epidural".

There is a growing body of literature supporting the use of PIEB. Programmed intermittent epidural bolus with PCEA compared with CEI with PCEA leads to higher maternal satisfaction, lower total LA consumption, and shorter second stage of labour. ${ }^{6}$ In our survey, PIEB with PCEA was the most used technique. This method has been previously suggested to result in higher maternal satisfaction, fewer instrumented deliveries, and fewer adverse outcomes. ${ }^{5}$ The slight predominance of PIEBbased regimens in our current study may represent shifting practices; however, several respondents identified barriers to adopting PIEB. These were-in order of frequencycost, incompatibility with available pumps, and unavailability of appropriate low concentration LA solutions in their formularies. These barriers, in the setting of inferred research translation delay, appear to have led to a state of clinical equipoise between CEI and PIEB. Further large-scale trials are warranted to elucidate any relative advantages and disadvantages of these techniques.

This study has potential limitations. We chose to survey institutions with obstetric anesthesiology fellowship programs, both to obtain data on leading academic practices in the training of future obstetric anesthesiologists and with hope that these groups would have a higher level of engagement and thence response rate. We appreciate that this selection bias does not allow for extrapolation to national practices in the USA or Canada. Surveys of a wider cross-section of practices are required to allow generalizability. We acknowledge that our limited sample size hinders our ability to draw statistical generalizations about nuanced trends such as bolus volume selection after DPE, or "next bolus" timing with different CSE dosing.

The survey provides informative contemporary data related to leading obstetric anesthesia institutions' labour epidural practices. Our data show areas of consensus and variability, and future surveys are needed to evaluate changes over time with evolving knowledge and studies. We hope our findings will encourage further exploration evidence-based practices in labour analgesia and provide a benchmark for providers seeking to establish or change practice patterns in their institution.
Author contributions Elliott Callahan contributed to study conception and design; acquisition, analysis, and interpretation of data; and drafting the article. Peter Yeh contributed to study conception and design; analysis, and interpretation of data; and drafting the article. Brendan Carvalho contributed to study conception and design, interpretation of data, and drafting the article. Ronald B. George contributed to all aspects of this manuscript, including study conception and design; acquisition, analysis, and interpretation of data; and drafting the article.

Disclosures Dr. George is a consultant for an Octapharma USA, Inc. Advisory Board and an Associate Editor of the Canadian Journal of Anesthesia.

Funding statement None.

Editorial responsibility This submission was handled by Dr. Sheila Riazi, Associate Editor, Canadian Journal of Anesthesia/Journal canadien d'anesthésie.

Open Access This article is licensed under a Creative Commons Attribution-NonCommercial 4.0 International License, which permits any non-commercial use, sharing, adaptation, distribution and reproduction in any medium or format, as long as you give appropriate credit to the original author(s) and the source, provide a link to the Creative Commons licence, and indicate if changes were made. The images or other third party material in this article are included in the article's Creative Commons licence, unless indicated otherwise in a credit line to the material. If material is not included in the article's Creative Commons licence and your intended use is not permitted by statutory regulation or exceeds the permitted use, you will need to obtain permission directly from the copyright holder. To view a copy of this licence, visit http://creativecommons.org/licenses/ by-nc/4.0/.

\section{References}

1. Jones L, Othman M, Dowswell, T, et al. Pain management for women in labour: an overview of systematic reviews. Cochrane Database Syst Rev 2012; DOI: https://doi.org/10.1002/14651858. CD009234.pub2.

2. Simmons SW, Cyna AM, Dennis AT, Hughes D. Combined spinalepidural versus epidural analgesia in labour. Cochrane Database Syst Rev 2007; DOI: https://doi.org/10.1002/14651858. CD003401.pub2.

3. van der Vyver $M$, Halpern $S$, Joseph $G$. Patient-controlled epidural analgesia versus continuous infusion for labour analgesia: A metaanalysis. Br J Anaesth 2002; 89: 459-65.

4. Carvalho B, George RB, Cobb B, McKenzie C, Riley ET. Implementation of programmed intermittent epidural bolus for the maintenance of labor analgesia. Anesth Analg 2016; 123: 965-71.

5. Хи J, Zhou J, Xiao H, et al. A systematic review and meta-analysis comparing programmed intermittent bolus and continuous infusion as the background infusion for parturient-controlled epidural analgesia. Sci Rep 2019; DOI: https://doi.org/10.1038/s41598019-39248-5.

6. George RB, Allen TK, Habib AS. Intermittent epidural bolus compared with continuous epidural infusions for labor analgesia: a systematic review and meta-analysis. Anesth Analg 2013; 116: 133-44. 